\title{
Multimodal imaging and high- throughput image-processing for drug screening on living organisms on-chip
}

\author{
Daniel Migliozzi \\ Matteo Cornaglia \\ Laurent Mouchiroud \\ Virginie Uhlmann \\ Michael A. Unser \\ Johan Auwerx \\ Martin A. M. Gijs
}




\title{
Multimodal imaging and high-throughput image-processing for drug screening on living organisms on-chip
}

\author{
Daniel Migliozzi, ${ }^{a}$ Matteo Cornaglia, ${ }^{a}$ Laurent Mouchiroud, ${ }^{b}$ Virginie Uhlmann, ${ }^{c}$ Michael A. Unser, ${ }^{c}$ \\ Johan Auwerx, ${ }^{b}$ and Martin A. M. Gijs $s^{a, \star}$ \\ ${ }^{a}$ Ecole Polytechnique Fédérale de Lausanne (EPFL), Laboratory of Microsystems, Lausanne, Switzerland \\ ${ }^{b}$ Ecole Polytechnique Fédérale de Lausanne (EPFL), Laboratory of Integrative Systems Physiology, Lausanne, Switzerland \\ 'Ecole Polytechnique Fédérale de Lausanne (EPFL), Laboratory of Biomedical Imaging, Lausanne, Switzerland
}

\begin{abstract}
A major step for the validation of medical drugs is the screening on whole organisms, which gives the systemic information that is missing when using cellular models. Caenorhabditis elegans is a soil worm that catches the interest of researchers who study systemic physiopathology (e.g., metabolic and neurodegenerative diseases) because: (1) its large genetic homology with humans supports translational analysis; (2) worms are much easier to handle and grow in large amounts compared with rodents, for which (3) the costs and (4) the ethical concerns are substantial. Here, we demonstrate how multimodal optical imaging on such an organism can provide high-content information relevant to the drug development pipeline (e.g., mode-of-action identification, dose-response analysis), especially when combined with on-chip multiplexing capability. After designing a microfluidic array to select small separated populations of $C$. elegans, we combine fluorescence and brightfield imaging along with high-throughput feature recognition and signal detection to enable the identification of the mode-of-action of an antibiotic. For this purpose, we use a genetically encoded fluorescence reporter of mitochondrial stress, which we studied in living specimens during their entire development. Furthermore, we demonstrate real-time, very large field-of-view capability on multiplexed motility assays for the assessment of the dose-response relation of an anesthetic. () The Authors. Published by SPIE under a Creative Commons Attribution 4.0 Unported License. Distribution or reproduction of this work in whole or in part requires full attribution of the original publication, including its DOI. [DOI: 10.1117/1.JBO.24.2.021205]
\end{abstract}

Keywords: multimodal imaging; fluorescence microscopy; image segmentation; high-content screening; microfluidic array; physiopathology.

Paper 180160SSPR received Mar. 15, 2018; accepted for publication Nov. 2, 2018; published online Nov. $27,2018$.

\section{Introduction: Optical Imaging of Caenorhabditis elegans in Microfluidic Chips}

To study human physiology, researchers often have only access to cells or tissues, which are unable to give complete information about the organism as a whole. To get insight of systemic responses to treatments, scientists, therefore, perform studies on entire organisms, such as mice, rats, or monkeys. In this case, ethical concerns rise and, from a practical point of view, these animals are expensive and their handling is labor-intensive. Thus, these studies cannot involve a high number of specimens, which prevents any high-throughput experimentation. Among the organisms of interest that could compensate these aspects, Caenorhabditis elegans is a soil worm of very little size $(\approx 1 \mathrm{~mm})$ that possesses many features to be employed as a model in compound screening: (i) it is small and simple to grow at little expenses, (ii) the current ethical concerns associated with it are much less restrictive than with rodents, (iii) imaging is straightforward thanks to its transparency, (iv) many orthologues of human genes are known in its genome, ${ }^{1}$ (v) its genetic manipulation is readily accessible and mastered (e.g., siRNA ${ }^{2}$ ), (vi) its developmental stages are well known and characterized, ${ }^{3}$ and (vii) many data on its physiology

*Address all correspondence to: Martin A. M. Gijs, E-mail: martin.gijs @epfl.ch are reported in curated databases. ${ }^{4,5}$ All these aspects make C. elegans a suitable model organism for high-content studies of systemic physiopathology ${ }^{6}$ when looking for candidate drugs $^{7}$ or targets, ${ }^{8}$ while maintaining the throughput facet. ${ }^{9}$ Crucial findings have already been obtained using $C$. elegans to develop therapeutic strategies for, among others, Huntigton's disease $^{10}$ and molecular inhibitors of $\beta$-amyloid aggregation in Alzheimer's disease. ${ }^{11}$

The development of assays to optically image and manipulate nematodes on chip has grown fast in the last decade. ${ }^{12}$ For instance, high-resolution fluorescence imaging was achieved for the study of neuronal synapsies ${ }^{13}$ and protein aggregation in muscles ${ }^{14}$ during nematode development on-chip. Using patterned microfluidic chambers and channels to isolate worm specimens on chip, bright-field imaging has been exploited for development ${ }^{15}$ and movement analysis during aging, ${ }^{16}$ chemical treatment, ${ }^{17}$ or electrical stimulation[gupta]. Localized optogenetic stimulation of chemosensory neurons was performed thanks to precise confinement of $C$. elegans specimens in narrow channels. ${ }^{18}$ However, as organisms can give large amounts of systemic information, combination of such modalities in a chip with multiplexing capabilities would be extremely advantageous for high-content screenings in drug development. ${ }^{19}$ For this purpose, we demonstrate here how multimodal imaging on a well-designed microfluidic chip for multiplexed analysis on $C$. elegans can provide quantitative 
findings on some crucial steps of the drug validation pipeline, such as mode-of-action identification and dose-response analysis.

\section{Methods}

\subsection{C. elegans Strain and Culture}

C. elegans strain was cultured at $20^{\circ} \mathrm{C}$ on nematode growth media (NGM) 90-mm Petri dishes seeded with the Escherichia coli strain OP50 (RRID = WB-STRAIN:OP50). The strains used in this study were wild-type Bristol N2, for the tetramisol study, and SJ4100 (zcIs13[hsp-6::GFP]), for the doxycyclin study, and were provided by the Caenorhabditis Genetics Center (University of Minnesota). Worms were suspended in S-medium solution prior to each microfluidic experiment.

\subsection{Image Acquisition and Processing}

For the mode-of-action study, we used an inverted microscope (Axio Observer, Zeiss) equipped with a $5 \times$ objective and two illumination systems: (i) a precisExcite High-Power LED illumination system (Visitron, Puchheim, Germany) for brightfield imaging and (ii) a Lambda DG4 illumination system (Sutter Instruments, Novato, California) for fluorescence imaging. The microscope had a motorized $x-y$-stage and the automated imaging process was controlled using VisiView Premier Image acquisition software (Visitron, Puchheim, Germany). Images were acquired with a Hamamatsu Orca-ER CCD camera (Hamamatsu, Solothurn, Switzerland). Bright-field and fluorescence exposure times were of 2 and $5 \mathrm{~ms}$, respectively. A sequence of pictures ( 2 per chamber) was taken every $20 \mathrm{~min}$. Image processing was performed with Fiji software. ${ }^{20}$ For the motility analysis, the same bright-field system was used, and images were recorded at a frame rate of $400 \mathrm{fpm}$. For doseresponse analysis, we used a stereomicroscope (SteREO Discovery.V8, Zeiss, Germany) coupled with an $0.5 \times$ objective to image the entire chip at once. Back-illumination had a ring shape to enable contrast enhancement in a dark-field mode. Images were acquired with a Hamamatsu Orca-ER CCD camera (Hamamatsu, Solothurn, Switzerland). Exposure time was $10 \mathrm{~ms}$. Images were recorded every $250 \mathrm{~ms}$. Images with very large field-of-view were analyzed manually, whereas for real-time movement tracking, we retrieved the medial axis of each $C$. elegans individual using a custom-open spline-based active contour algorithm. ${ }^{21}$ Active contours consist of a flexible curve that automatically deforms from an initial position in the image toward its target, which is here the medial axis of each nematode. The curve deformation is the result of the minimization process on an appropriately designed cost function. Our active contours are constructed using a Hermite-based model ${ }^{22}$ with six control points to define the curve. Their associated cost is a combination of texture-based and ridge information that allow identifying candidates nematodes. Ridge detection is carried out with steerable filters, ${ }^{23}$ which also provide local orientation information. The latter increases robustness in a medial axis search in situations where several C. elegans are in close contact, which happens regularly in our data. This spline active contour construction provides an efficient and compact representation of each $C$. elegans: continuous medial axes are constructed from few parameters and allow extracting measurements such as length and curvature at subpixel resolution. The resulting semiautomated image analysis pipeline is implemented in Fiji. ${ }^{20}$ The location of nematode extremities is specified by the user at each frame and is sufficient for the active contours to automatically adapt and find the medial axis of each individual. Thanks to a high-temporal resolution, tracking is performed by propagating medial axes from previous frames to the next and reoptimizing the active contours to fit the new image content.

\subsection{Silicon Master Fabrication and PDMS Chip Preparation}

Microfluidic devices were prepared by soft lithography. Briefly, after surface activation (20 min in hexamethyldisilazane vapor) of 4 in Si-wafers, we spin-coated $4 \mu \mathrm{m}$ of positive photoresist (AZ9260). We then exposed in a hard-contact mode for $16 \mathrm{~s}$ $\left(160 \mathrm{~mJ} / \mathrm{cm}^{2}\right)$ and developed with 115 -s contact time. After back-side rinsing, we obtained fine structures with $1-\mu \mathrm{m}$ resolution. Dry etching was achieved with the standard $\mathrm{BOSCH}$ process at $25^{\circ} \mathrm{C}$, with process time adjusted to obtain the desired height of the structure (etching rate $\approx 3.5 \mu \mathrm{m} / \mathrm{min}$ ). Polydimethylsiloxane (PDMS) molding mixture of 10:1 (prepolymer:curing agent) was mixed manually and degassed for $1 \mathrm{~h}$. The mixture obtained was then poured onto a Si-master or spin-coated on a $\mathrm{Si}$ master or PDMS slab depending on the desired structure. Curing was achieved by keeping at $100^{\circ} \mathrm{C}$ for 1 to $2 \mathrm{~h}$. The solid PDMS obtained was then peeled off and cleaned with the following procedure: rinsing with water, 6-min sonication in ethanol, rinsing with deionized water, drying with air-gun. The PDMS chip and its support (a glass slide or another PDMS chip depending on the desired structure $)$ were then plasma-activated $\left(0.2 \mathrm{mbar}_{2}, 3.0 \mathrm{~mA}\right.$, $0.5 \mathrm{kV}$ ) for $70 \mathrm{~s}$ and bonded together.

\subsection{Microfluidic Calculations}

When one considers a pressure-driven flow in a thin channel (width $=w \ll h=$ height), the ratio of the flow rate $Q$ of the liquid to the pressure difference $\Delta P$ causing this flow is the hydraulic resistance and is shown to depend on the geometrical features of the channel as follows: ${ }^{24} \frac{\Delta P}{Q}=R \approx \frac{12 \mu L}{w h^{3}(1-0.63 h / w)}$, where $L$ is the length of the channel and $\mu$ is the viscosity of the liquid. For the same pressure difference, the higher the resistance, the lower the flow rate. The Reynolds number is defined as $R e=\frac{\rho v d}{\mu}$, where $\rho$ is the density of the fluid, $v$ is the flow rate, and $d$ is the hydraulic diameter (i.e., the size of the channel). $R e$ represents the ratio of the inertial to the viscous forces. For our conditions $(d<1 \mathrm{~mm}, v<1 \mu \mathrm{L} / \mathrm{s})$ $R e<10^{-6}$, and the inertial forces are negligible. This means, for instance, that the liquids are very stable when changing flow direction or passing around an obstacle. This is particularly useful to well control the flow rate in channels with different shapes and containing obstacles such the one we have used for this project. The Péclet number is defined as $P e=\frac{v d}{D}$, where $D$ is the diffusion coefficient of the molecules transported by the fluid. $P e$ represents the ratio of the mass transport due to advection to the one due to diffusion. This value is very high $\left(P e>10^{4}\right)$ in our conditions $\left(D \approx 10^{-9} \mathrm{~m}^{2} / \mathrm{s}\right.$ for small chemicals), which means that the concentration of a chemical species present in the liquid will change mainly by advection.

\subsection{Feeding and Drug Administration On-Chip}

E. coli (for feeding) and drug (for screening) administration were performed in a continuous-perfusion mode by withdrawing 
liquid from the outlet at $>4 \mathrm{~nL} / \mathrm{s}$, with periodic washing-andrenewing cycles ( 1 per hour) by increasing up to $30 \mathrm{~nL} / \mathrm{s}$ in $1 \mathrm{~min}$ and decreasing down to $4 \mathrm{~nL} / \mathrm{s}$ in $1 \mathrm{~min}$.

\section{Results}

\subsection{Microfluidic Array for Drug Screening on C. elegans}

\subsubsection{Chip design}

We designed a microfluidic chip to perform all the following steps: select worm specimens into separated chambers, make them grow from the larval stage to the adult age, and administrate drugs at desired time during the nematode lifespan. The final design of the chip is shown in Fig. 1. It consists of several long channels divided into chambers by small filters consisting of PDMS posts. Each inlet is meant to be plugged into a reservoir, and the outlet into a syringe. This design is almost completely symmetric to ensure maximum homogeneity in the fluid dynamics in the different paths, which increases the control of the flow rate. We have optimized the geometrical features of the filtering channels to select larval stages of $C$. elegans and make them trapped into the chambers.

Our microfluidic chip is designed to directly select larval specimens from a mixed population. We inject a mixed population from the outlet of the chip until we have the desired amount of worms before the first rank of filters; then we inject the liquid with pulses of 1 to $2 \mathrm{~s}$ at 10 to $20 \mu \mathrm{L} / \mathrm{s}$. The pulses at very high flow rate make the small channels between the flexible posts expand to allow the larvae to pass-through. This is enabled by the hydraulic compliance of our PDMS posts, which are compressed during the pulses because of their aspect ratio (100- $\mu \mathrm{m}$ high, $70-\mu \mathrm{m}$ wide) and go back to their shape when the flow stops. After 10 to 20 pulses, the chambers become filled with different populations of the first larval stage, which can

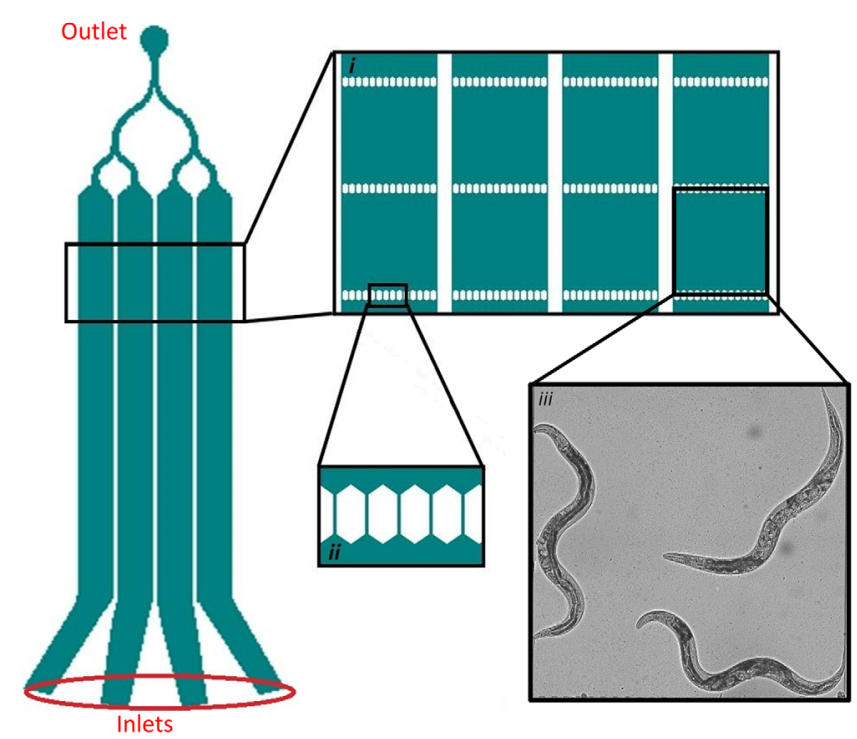

Fig. 1 Design of the chip for worm isolation and multiplexed analysis. Schematic of the microfluidic chip with a single outlet and several inlets and chambers for multiplexed analysis. The worm chambers (i) are separated by small channels (ii) of $100-\mu \mathrm{m}$ length and 8 - to $12-\mu \mathrm{m}$ width. The height of the structure is $100 \mu \mathrm{m}$. Worms are separated into small populations ( 1 to 5 specimens) during their entire lifespan (iii). develop while remaining isolated from each other (Fig. 1). This protocol serves to isolate larvae from a mixed population, and gives a much higher screening resolution ( 1 to 5 worms per chamber) with respect to standard tests on agar plates. The trapped worms cannot escape from the chamber by swimming, and for flow rates $<200 \mathrm{~nL} / \mathrm{s}$, the PDMS post do not deform enough to enable worm passage. This is, therefore, an upper limit for the flow rate to be used for feeding and for drug administration. When the adult worms lay eggs, which subsequently hatch, the same pulsating flow protocol can be used to wash away the new larvae, so that always the same worms are kept in the chambers from the beginning till the end of their lifecycle eventually.

\subsubsection{Characterization of cross-mixing between channels}

As, for this simple geometry, the Reynolds number and the Peclét number (Sec. 2.4) are very low and very high, respectively, the mass transport occurs mainly by advection. To avoid the cross contamination of the different channels, the diffusion and the flow of the drug from a channel to another one must be minimized. For this, we have an imposed flow rate for several fluidic paths using only one syringe, in a very straightforward implementation. Finite-element simulations show that the threshold value for the outflow to prevent cross-contamination should be between 1 and $10 \mathrm{~nL} / \mathrm{s}$ (Sec. 5). To verify this theoretical value, we tested the cross-mixing behavior experimentally using inks of different colors on chips with four inlets. We plugged each inlet in a reservoir containing the ink [Fig. 2(a)], and withdrew liquid with a syringe from the outlet at several flow rates. Results are shown in Figs. 2(b) and 2(c). Colored lines are well distinguishable and do not cross contaminate. We can conclude that for a total outflow of $4 \mathrm{~nL} / \mathrm{s}$ (corresponding at about $1 \mathrm{~nL} / \mathrm{s}$ flowing in each of the four channels), there is no back diffusion of the fluid from the different paths, which is in accordance with the simulated fluidic behavior. To further quantify the diffusion phenomenon on the chip, we studied the behavior of the ink diffusion after completely stopping the flow. We recorded pictures at a different time after stopping a $4 \mathrm{~nL} / \mathrm{s}$ outflow [Fig. 2(d)]. We observed that the diffusion is very slow and that it takes about $1 \mathrm{~h}$ to have a visible change in ink concentration in the first chamber of the adjacent channel. To evaluate the flow rate of the individual channels relative to the total outflow imposed, we measured the time to empty $300-\mu \mathrm{L}$ wells for each channel: these were found to be within a $6 \%$ range, which guarantees very similar flow from all the channels.

\subsection{Multimodal Imaging on Proof-of-Concept Screens}

\subsubsection{Combined bright-field and fluorescence imaging for mode-of-action identification: study of an antibiotic}

To demonstrate the possibility of long-term mode-of-action analysis, we designed a proof-of-concept fluorescence-reporting assay on doxycycline, an antibiotic known to induce mitochondrial stress. ${ }^{25}$ Expression of the heat-shock protein 6 (HSP-6) is induced in the presence of mitochondrial stress, ${ }^{26}$ thus we used a C. elegans strain that carried the GFP-gene under the control of the HSP-6 promoter, and the goal was to monitor the growth and the GFP expression of the worms during their development. We treated a part of the worms with doxycycline (treated group), 
(a)

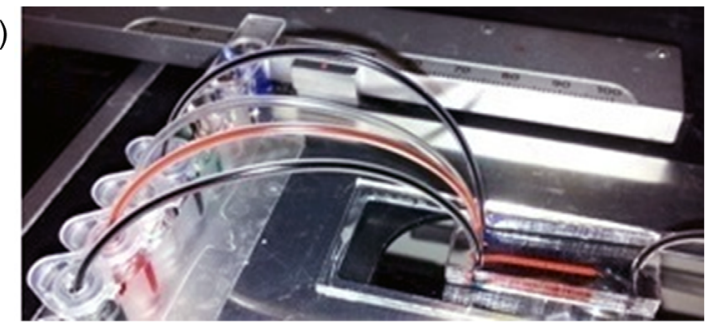

(b)

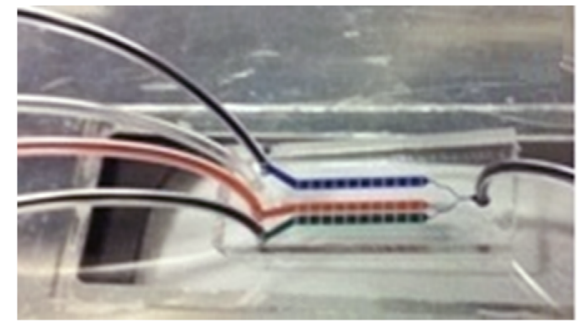

(c)

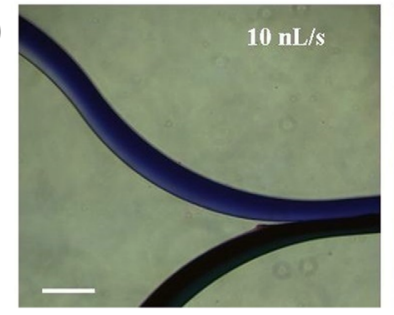

(d)
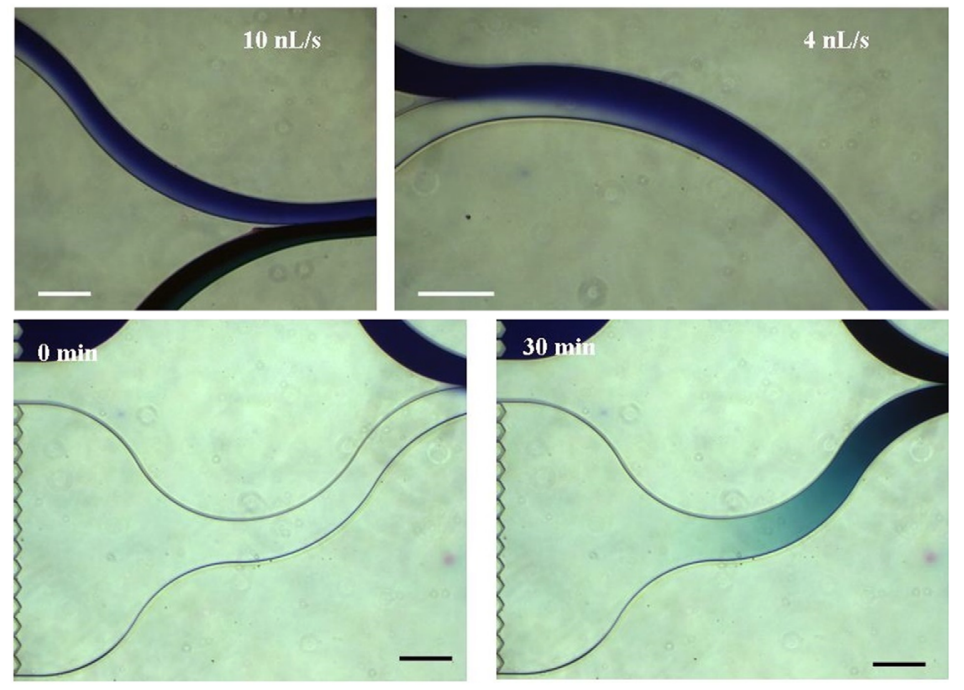

Fig. 2 Analysis of the cross-mixing between channels. (a) Inlets plugged into a $200-\mu L$-well-strip, filled with inks. (b) Top-view of the chip during withdrawal of liquid from the outlet at $100 \mathrm{~nL} / \mathrm{s}$. (c) Bright-field images of the outlet part of the chip, showing the interfaces between two fluidic paths for the indicated value of the total outflow. (d) Bright-field images of the part of the chip closest to the oulet, at different time after stopping a $4 \mathrm{~nL} / \mathrm{s}$ outflow. Scale bars: $200 \mu \mathrm{m}$.

and treated the other worms with vehicle (control group). Both groups of worms were on the same chip and were treated and imaged at the same time every $20 \mathrm{~min}$ for $52 \mathrm{~h}$.

Such screens resulted in more than 3000 images, thus, to quantify worm growth and GFP expression for all of them in a robust and automatic fashion, we have developed an image-processing algorithm that runs image analysis on the entire set of pictures. The image treatment aimed at obtaining quantitative information on the developmental stage of the worms (from bright-field images), and on their GFP expression (from fluorescence images).

The analysis of the bright-field images used the contrast between the dark border of the worms and the bright surroundings to segment the nematodes in each image:

1. A gray-scale opening was applied to smooth objects and remove small objects (e.g., dust, isolated bacteria).

2. A minimum-filter was applied to enlarge dark objects such as the border of the worms.

3. A threshold was applied to select the dark objects only.

4. A binary-opening was applied to select only largeenough objects.

5. The boundary length of these objects was measured.

6. The average perimeter of the worm was considered as being the previous value divided by the number of worms in the image.
The analysis of the fluorescence images used the contrast between the fluorescent body of the worms and the dark background to quantify only the signal coming from the nematodes in each image:

1. A "median" image was calculated by taking the pixelby-pixel median gray value along the axis of time and was subtracted from each fluorescence image to set the background to almost zero.

2. For each image:

(a) A Gaussian filter (2-pixel radius) was applied to eliminate the small isolated bright objects and to reduce the noise.

(b) A threshold was applied: the pixels above the threshold were considered as being the signal, and the ones below the threshold were considered as being the background.

This method provides an effective way to estimate the growth and the GFP expression during the entire lifecycle. Figure 3 shows the performance of this procedure in recognizing the worms in the bright-field images, and in distinguishing the signal from the background in the fluorescence images (i.e., the noise is highly reduced and the background subtracted). We used the contrast-to-noise ratio (CNR) to evaluate the quality of the fluorescence detection: $\mathrm{CNR}=\frac{\bar{S}-\bar{B}}{\Delta B}$, where $\bar{S}$ and $\bar{B}$ are the mean of the signal and the background, and $\Delta B$ is the standard deviation of the background; CNR $>3$ means very high 


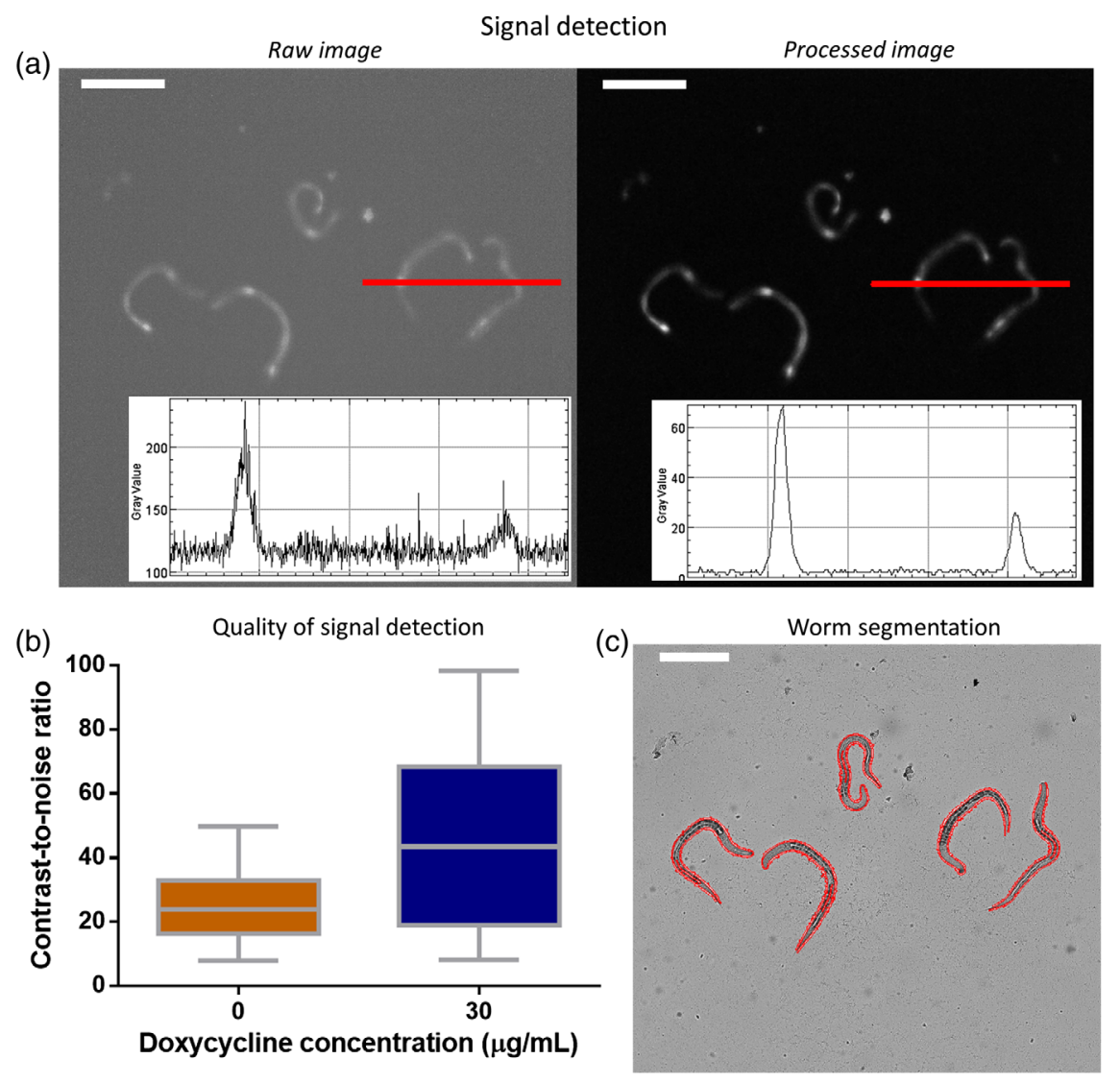

Fig. 3 High-throughput image-processing for signal detection and worm recognition. (a) Fluorescence image before (left) and after (right) treatment. The insets show the intensity profile along the red lines in the main images. (b) Tukey box-plot of the contrast-to-noise ratio resulting from the fluorescence detection algorithm. (c) Bright-field image where the worms are outlined in red after automatic recognition of their border. Scale bars: $200 \mu \mathrm{m}$.

separation between signal and background. Contrast-to-noise ratio for the fluorescence detection was $>10$ for all the conditions, with central tendency between 20 and 40 for treated and untreated conditions (Fig. 3).

Worms that were treated with doxycycline showed slower growth and increased GFP expression compared with untreated worms (Fig. 4). These results indicate that a higher mitochondial stress may affect and delay the development process.

\subsubsection{Combined real-time imaging and single-worm tracking for dose-response assessment and motility analysis: study of an anesthetic}

To illustrate the advantage of having (i) few nematode specimens in a chamber, (ii) many chambers to increase the statistics, and (iii) several channels to test different conditions, we performed a motility assay in real time and with a very large field of view (up to $\approx 1 \times 1.4 \mathrm{~cm}^{2}$ ). We used several concentrations $(0,5,50,500,625,1250,2500$, and $5000 \mu \mathrm{M})$ of tetramisole, an anesthetic. The aim of the assay was to correlate the concentration of the drug with the number of anesthetized worms to trace the dose-response curve for this drug. We injected larval specimens of $C$. elegans into the chip as described in Sec. 3.1.1, fed them for 2 days to obtain adult worms, and then we administrated the drug for $40 \mathrm{~min}$.

We imaged all the chambers at once using a stereomicroscope with a suitable dark-field illumination to enhance the contrast and make the adult worms appear much brighter than the background (Fig. 5). We subsequently analyzed the images directly on the recorded video: we counted the number of anesthetized worms per chamber every $10 \mathrm{~min}$ from the beginning of the drug administration for $40 \mathrm{~min}$, and then calculated the total percentage per channel $(1$ channel $=1$ concentration). As proposed by Qian et al., ${ }^{27}$ the worms were considered as being anesthetized when they displayed coiling or no movement at all. We traced the dose-response curve for this drug using the value of the mean percentage of anesthetized worms at 30 and $40 \mathrm{~min}$ after tetramisole injection (Fig. 5). By fitting the data with a sigmoid function $y=$ $\frac{100}{1+10^{H} \text { slope }^{\left(E C_{50}-x\right)}}$, we obtained an $\mathrm{EC}_{50}=72 \mu \mathrm{M}$ (i.e., the effective concentration that cause half of the total effect) and a Hill-slope $=0.95 \mu \mathrm{M}^{-1}$ (i.e., the slope of the curve at $\mathrm{EC}_{50}$ ).

To characterize the paralysis of the nematodes, we have studied their swimming behavior during the administration of tetramisole $(200 \mu \mathrm{M})$. We tracked the medial axis of each worm over time using semiautomatic recognition of worm body based on splines. ${ }^{22}$ To describe the swimming movement, we measured the length of the medial axis $(L)$ and the distance between its extremities $(d)$, and we defined the straightness of the worms as $d / L$ : which is close to 1 for a straight worm, and decreases when the worm displays coiling. The variation of the straightness over time provides information on the nematode swimming. The results of this analysis are shown in Fig. 6. 
(a)
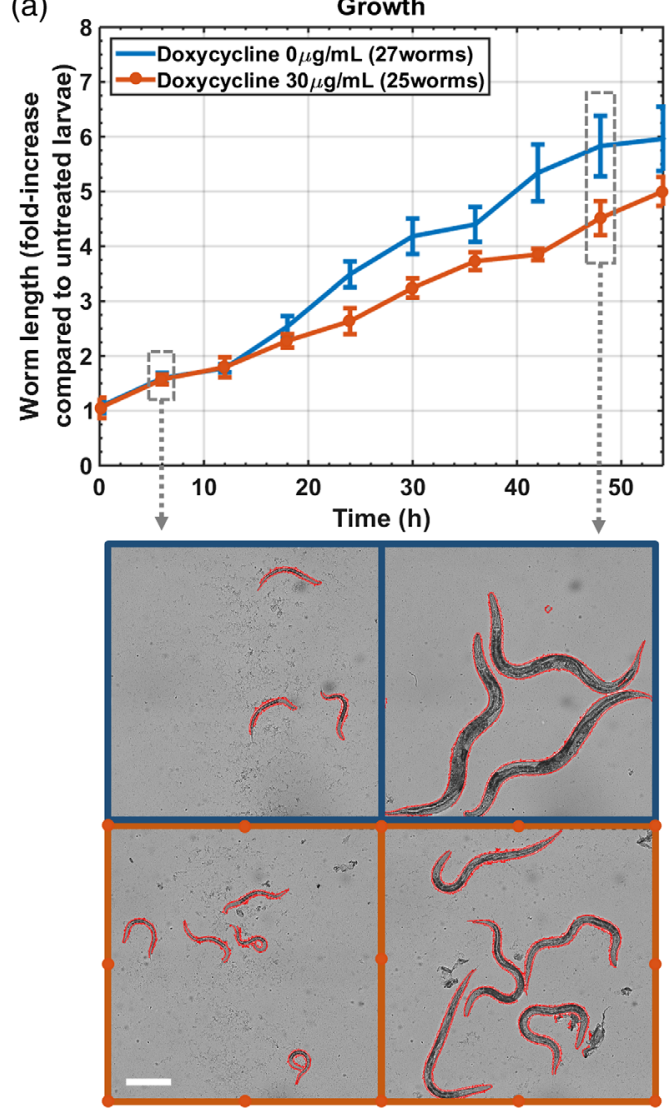

(b)
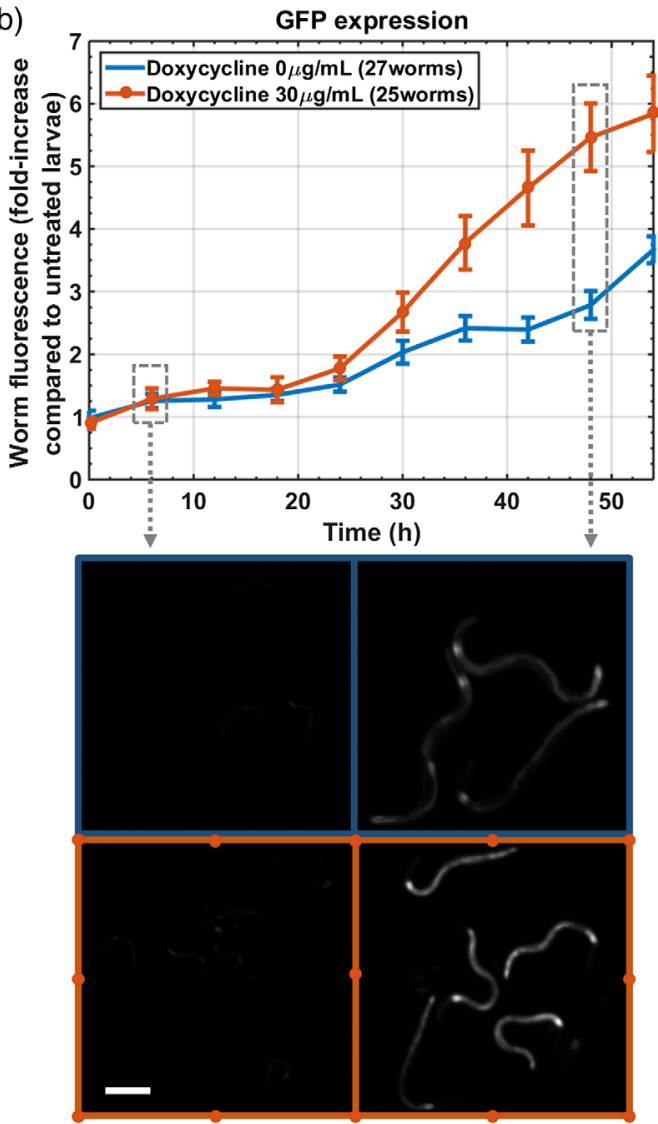

Fig. 4 Combined bright-field and fluorescence imaging for mode-of-action identification. (a) Worm length over time for worms untreated/treated with doxycycline. (b) Worm fluorescence over time for worms untreated/treated with doxycycline. Below each graph are examples of treated images for different conditions. The fold-increase is calculated by taking the untreated larvae as the normalization unit. Data are shown as mean \pm SEM from five different chambers for three neighboring time-points. Scale bars: $200 \mu \mathrm{m}$.

(a)

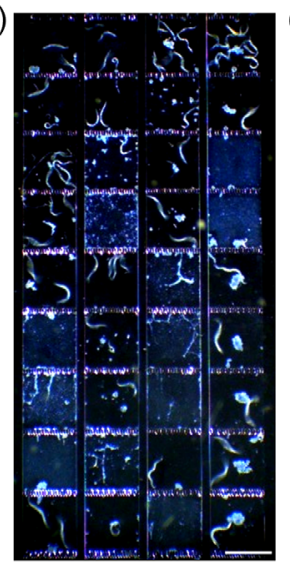

(b)

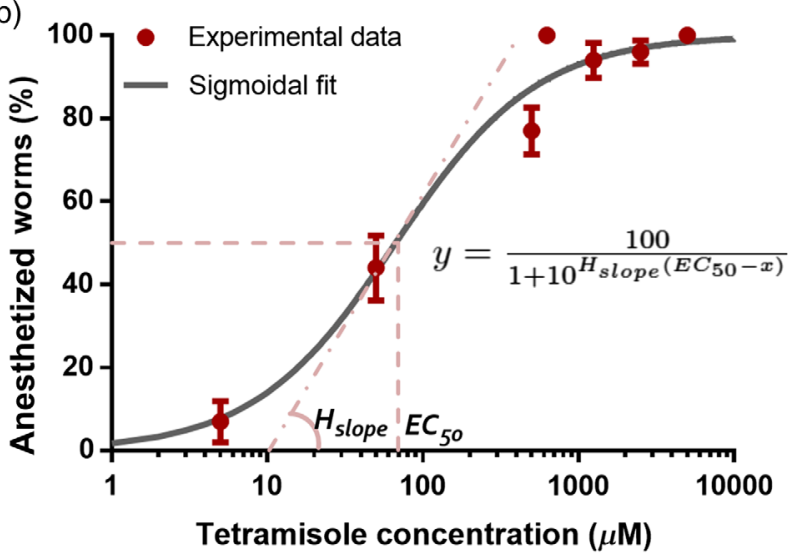

Fig. 5 Motility analysis with real-time, dark-field, large field-of-view imaging. (a) Image of the chip through a stereomicroscope in dark-field illumination. Each channel was used to test a different concentration. Scale bar: $1 \mathrm{~mm}$. (b) Dose-response curve for tetramisole; data are plotted as mean $\pm \mathrm{SEM}$ of the anesthetized worms after 30 and 40 min of tetramisole administration. The solid line indicates a sigmoidal fit. The dashed and dashed-and-dotted lines indicate the values for the $50 \%$ effective concentration $\left(\mathrm{EC}_{50}\right)$ and the hill-slope $\left(H_{\text {slope }}\right)$, respectively.

Before complete paralysis, the worms display a transition phase during which fast variations of their straightness occur due to a highly accentuated coiling behavior, which confirms previous observations on a similar compound. ${ }^{27}$ The slight delay between the behavior of different specimens may be due to individual-specific sensitivity to the compound tested, and demonstrate how the single-worm resolution can disclose subtle phenotypes. 

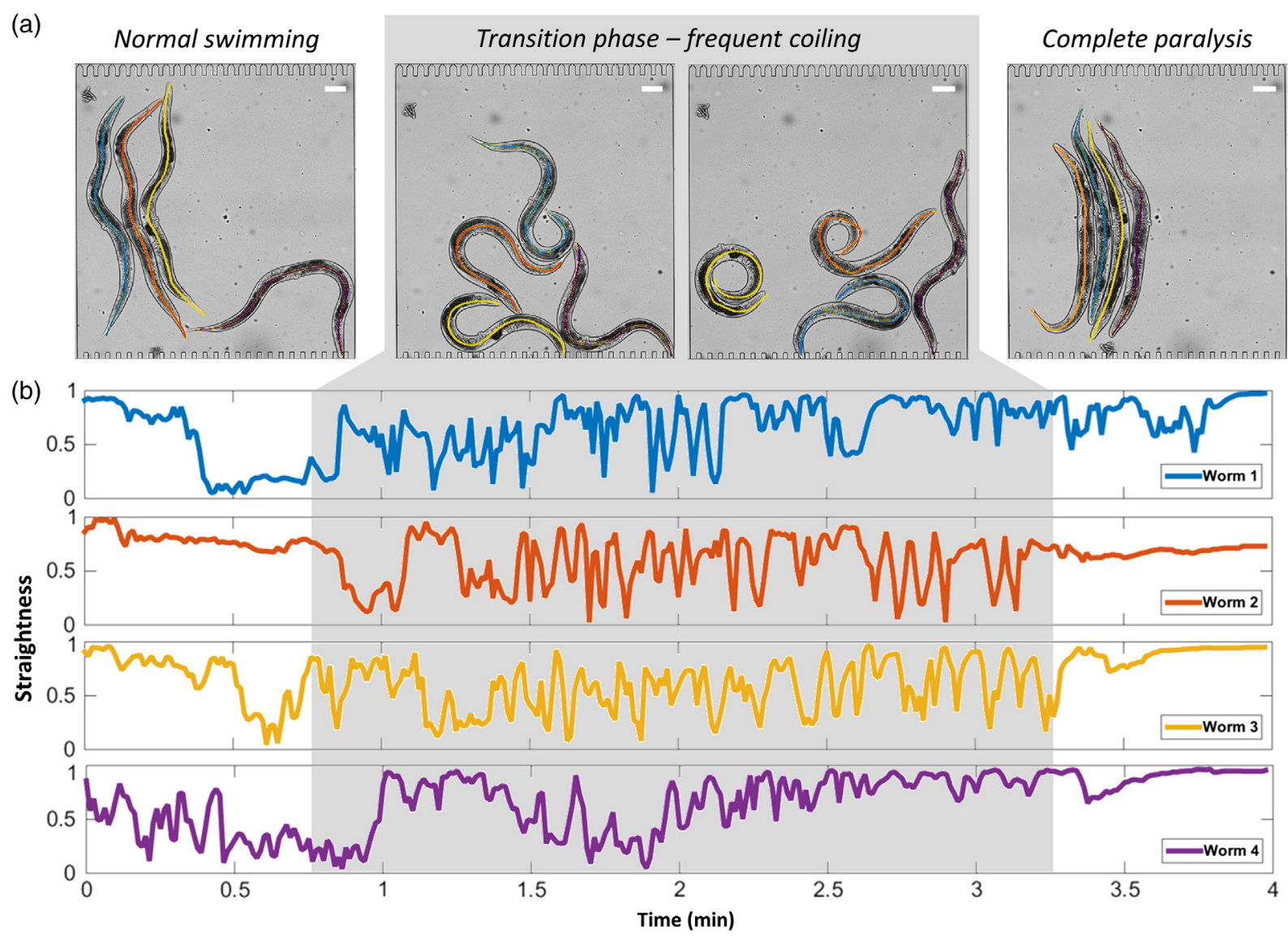

Fig. 6 Real-time tracking of nematode movement at a single-worm resolution. (a) Bright-field images of worms during administration of tetramisol $(200 \mu \mathrm{m})$. The medial axes are indicated in color. (b) Straightness of the worms showed in A over time. Scale bars: $10 \mu \mathrm{m}$.

\section{Discussion}

Studying whole organisms can result in deeper understanding of the systemic effects of drugs. C. elegans is a potential candidate for preclinical screening of bioactive compounds because of its large genetic homology with humans, as well as for its simplicity to be genetically modified, and grown in large quantities without expensive costs or ethical issues. Due to its transparency, live optical imaging on this organism is also particularly straightforward.

To enable high-content screens, we have performed multimodal optical imaging coupled with high-throughput imageprocessing on small populations of $C$. elegans specimens trapped in a microfluidic array. We demonstrated the opportunity to link a phenotypic effect (such as the arrest of growth during development) to an underlying molecular mechanism (such as the increased mitochondrial stress), which is very powerful when searching the biomolecular pathway causing a disease.

By taking advantage of the multiplexing capability of our microfluidic design, we also quantified the dose-response relation of an anesthetic with real time, large field-of-view, darkfield imaging of the entire chip at once, which highly increases the throughput of a step that is performed on any potential drug during the development pipeline, to test its effect and its toxicology.

Finally, we demonstrated the possibility to obtain subtle motility features at single-worm level, which can be used to study the direct effect of drugs targeting muscles or motor-neurons.
Levamisol, for instance, is an FDA-approved isomeric form of tetramisole, which acts as a nicotinic acetylcholine receptor agonist on worm muscles, leading to paralysis. $^{28}$

In terms of throughput, the current design can be scaled up and up to 80 chambers can be imaged at the same time with a stereomicroscope when resolution is not a requirement for the assay. However, for higher-resolution imaging, only 1 chamber should be imaged at each time. This matches and, in some cases, even outrates the performance of previously reported platforms for optical analysis of worms in microfluidic chips. ${ }^{12-18}$

These results pave the way to systematic multimodal imaging of living organisms on chip to empower crucial steps of the drug development process, especially when combined with multiplexing microfluidic capability.

\section{Appendix A: Finite-Element Simulation of the Cross-Mixing on the Chip}

We simulated the flow pattern in the chip for several values of the total outflow at the outlet. We separated the problem in two steps:

1. Computation of the stationary velocity of the fluid for a given outflow. We assigned a zero initial condition for all the variables, imposed a constant outflow at the outlet and a constant pressure at the inlet.

2. Computation of the stationary concentration of a molecule loaded into one of the inlets at a given 

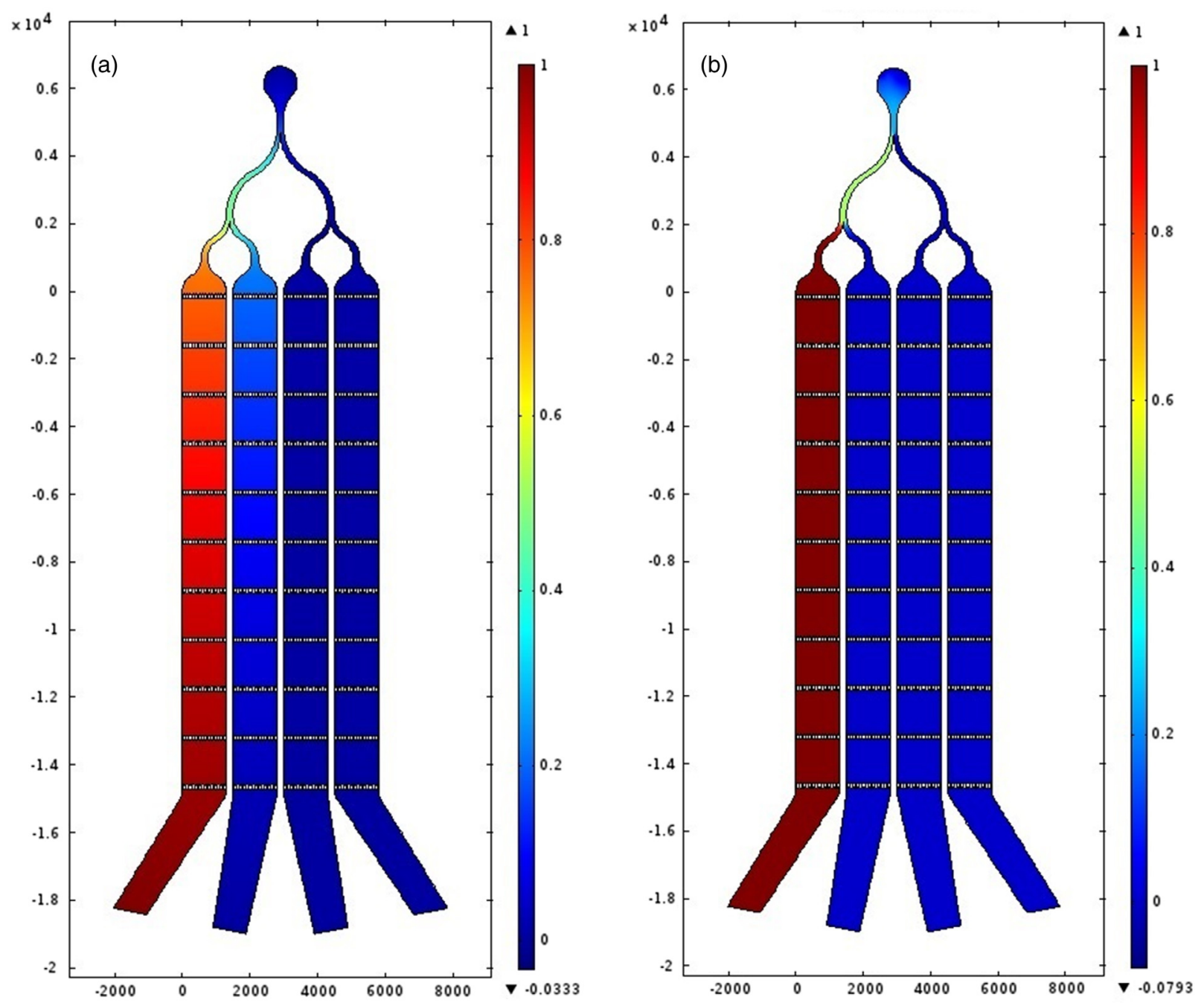

Fig. 7 Simulated cross mixing between channels. (a) Color-coded normalized concentration for a total outflow of $1 \mathrm{~nL} / \mathrm{s}$ at the unique outlet. (b) Color-coded normalized concentration for a total outflow of $10 \mathrm{~nL} / \mathrm{s}$ at the unique outlet. Diffusion coefficient was estimated as the one for small molecules: $10^{-6} \mathrm{~cm}^{2} / \mathrm{s}$. Length units are in micrometers.

concentration, given the velocity field obtained in the previous step. We assigned a zero initial condition for the concentration, four flow-driven inflows at the inlets (with $1 \mathrm{mM}$ at one of them and $0 \mathrm{mM}$ at the other), and an flow-driven outflow at the outlet.

We observe that, for an outflow of about $\mathrm{nL} / \mathrm{s}$, a small amount of molecule loaded onto a channel diffuses back into the adjacent channel, whereas at $10 \mathrm{~nL} / \mathrm{s}$ there is no crossmixing (Fig. 7).

\section{Disclosures}

M. A. M. G., M. C., and D. M. have an International Patent Application No. PCT/WO 2016/063199 A1, filed in October 20th 2014 as a Provisional Application No. PCT/IB214/ 065472, for a device that is related to this work.

\section{Acknowledgments}

This work was supported by the EU Ideas program (ERC-2012AdG-320404) and by the Gebert Ruf Stiftung (GRS-025/16).

\section{References}

1. D. D. Shaye and I. Greenwald, "OrthoList: a compendium of C. elegans genes with human orthologs," PLoS One 6(5), e20085 (2011).

2. R. L. Corrêa et al., "MicroRNA-directed siRNA biogenesis in Caenorhabditis elegans," PLoS Genet. 6(4), e1000903 (2010).

3. U. Deppe et al., "Cell lineages of the embryo of the nematode Caenorhabditis elegans," Proc. Natl. Acad. Sci. U. S. A. 75, 376-380 (1978).

4. Wormatlas, http://www.wormatlas.org/.

5. WormBook, http://www.wormbook.org/.

6. A. G. Alexander, V. Marfil, and C. Li, "Use of Caenorhabditis elegans as a model to study Alzheimer's disease and other neurodegenerative diseases," Front. Genet. 5, 279 (2014).

7. M. Artal-Sanz, L. de Jong, and N. Tavernarakis, "Caenorhabditis elegans: a versatile platform for drug discovery," Biotechnol. J. 1, 1405-1418 (2006).

8. T. Kaletta and M. O. Hengartner, "Finding function in novel targets: C. elegans as a model organism," Nat. Rev. Drug Discovery 5, 387-399 (2006).

9. L. P. O'Reilly et al., "C. elegans in high-throughput drug discovery," Adv. Drug Delivery Rev. 69-70, 247-253 (2014).

10. H. Jeong et al., "Acetylation targets mutant huntingtin to autophagosomes for degradation," Cell 137, 60-72 (2009).

11. J. Habchi et al., "Systematic development of small molecules to inhibit specific microscopic steps of ab42 aggregation in Alzheimer's disease," Proc. Natl. Acad. Sci. U. S. A. 114, E200-E208 (2017). 
12. N. A. Bakhtina and J. G. Korvink, "Microfluidic laboratories for C. elegans enhance fundamental studies in biology," RSC Adv. 4, 4691-4709 (2013).

13. J. Krajniak and H. Lu, "Long-term high-resolution imaging and culture of C. elegans in chip-gel hybrid microfluidic device for developmental studies," Lab Chip 10, 1862-1868 (2010).

14. M. Cornaglia et al., "Automated longitudinal monitoring of in vivo protein aggregation in neurodegenerative disease C. elegans models," Mol. Neurodegener. 11, 17 (2016).

15. M. C. Letizia et al., "A design of experiment approach for efficient multi-parametric drug testing using a Caenorhabditis elegans model," Integr. Biol. 10, 48-56 (2018).

16. S. E. Hulme et al., "Lifespan-on-a-chip: microfluidic chambers for performing lifelong observation of C. elegans," Lab Chip 10, 589-597 (2010).

17. K. Chung et al., "Microfluidic chamber arrays for whole-organism behavior-based chemical screening," Lab Chip 11, 3689-3697 (2011).

18. H. Lee et al., "A multi-channel device for high-density target-selective stimulation and long-term monitoring of cells and subcellular features in C. elegans," Lab Chip 14, 4513-4522 (2014).

19. D. Migliozzi et al., "A microfluidic array for high-content screening at whole-organism resolution," Proc. SPIE 10491, 104910D (2018).

20. J. Schindelin et al., "Fiji: an open-source platform for biological-image analysis," Nat. Methods 9, 676-682 (2012).

21. R. Delgado-Gonzalo et al., "Snakes on a plane: a perfect snap for bioimage analysis," IEEE Signal Process. Mag. 32, 41-48 (2015).

22. V. Uhlmann, J. Fageot, and M. Unser, "Hermite snakes with control of tangents," IEEE Trans. Image Process. 25, 2803-2816 (2016).

23. M. Jacob and M. Unser, "Design of steerable filters for feature detection using canny-like criteria," IEEE Trans. Pattern Anal. Mach. Intell. 26, 1007-1019 (2004).

24. A. G. G. Toh et al., "Engineering microfluidic concentration gradient generators for biological applications," Microfluid. Nanofluid. 16, 1-18 (2014).

25. R. H. Houtkooper et al., "Mitonuclear protein imbalance as a conserved longevity mechanism," Nature 497, 451-457 (2013).

26. T. Yoneda et al., "Compartment-specific perturbation of protein handling activates genes encoding mitochondrial chaperones," J. Cell Sci. 117, 4055-4066 (2004).

27. H. Qian et al., "Levamisole resistance resolved at the single-channel level in Caenorhabditis elegans," FASEB J. 22, 3247-3254 (2008).

28. J. A. Lewis et al., "Levamisole-resitant mutants of the nematode Caenorhabditis elegans appear to lack pharmacological acetylcholine receptors," Neuroscience 5, 967-989 (1980).

Daniel Migliozzi received his BSc degree in engineering physics from Politecnico di Milano (Italy) in 2011. He received his double MSc degree in bioengineering from EPFL (Switzerland) and Ecole Polytechnique (France) in 2014. He worked on research projects in collaboration with CEA-LETI (France), Nestlé and Lunaphore Technologies (Switzerland). Currently, he is a PhD candidate at EPFL, where he develops optical and microfluidic technologies for cancer diagnostics and live-cell imaging. He is a student member of SPIE.
Matteo Cornaglia received his MSc degree in nanotechnologies for ICT, as a joint study agreement between Politecnico di Torino (Italy), Institut National Polytechnique de Grenoble (France) and EPFL (Switzerland). He obtained his $\mathrm{PhD}$ in microsystems and microelectronics in 2015 with his thesis titled "Merging microfluidics and micro-array concepts: from molecular to nematode-based bioassays." $\mathrm{He}$ is cofounder of NAGI bioscience, which develops microfluidic technologies for the study of organisms on chip.

Laurent Mouchiroud received his MSc degree in molecular and cellular biology from the Université Claude Bernard (France) in 2006 and his $\mathrm{PhD}$ in biology from the same university in 2010 . He joined the Laboratory of Integrative Systems Physiology at EPFL (Switzerland) in 2010 as a postdoctoral scientist. His work includes the characterization of pathophysiological mechanisms in $C$. elegans models of diseases. He is a cofounder of NAGI Bioscience, which develops microfluidic technologies for the study of organisms on chip.

Virginie UhImann received her $\mathrm{PhD}$ in electrical engineering from EPFL (Switzerland) in 2017. Her research interests focus on bioimage informatics and include both a theoretical (continuous representations for image analysis) and an applied track (collaborative projects with biologists and software development). Currently, she is a postdoc researcher at the Biomedical Imaging Group of Prof. M. Unser at EPFL. She is an associate member of the Bioimaging and Signal Processing Technical Committee of IEEE Signal Processing Society.

Michael A. Unser received his MS and $\mathrm{PhD}$ degrees in electrical engineering in 1981 and 1984, respectively, from the Swiss Federal Institute of Technology (EPFL) in Lausanne, Switzerland. From 1985 to 1997, he was with the Biomedical Engineering and Instrumentation Program, National Institutes of Health, Bethesda, conducting research on bioimaging and heading the Image Processing Group. He has a strong interest in sampling theories, multiresolution algorithms, wavelets, and the use of splines for image processing.

Johan Auwerx received both his MD and PhD degrees in molecular endocrinology at the Katholieke Universiteit in Leuven, Belgium. He was a postdoctoral research fellow in the Departments of Medicine and Genetics of the University of Washington in Seattle. He is a professor at the EPFL (Switzerland), where he occupies the Nestle Chair in Energy Metabolism. He has been using molecular physiology and systems genetics to understand metabolism in health, aging, and disease.

Martin A. M. Gijs received his degree in physics from Katholieke Universiteit Leuven (Belgium) in 1981, and his PhD in physics at the same university in 1986. He joined the Philips Research Laboratories in Eindhoven (Netherlands) in 1987. He joined EPFL in 1997. His present interests are in developing technologies for novel magnetic devices, new microfabrication technologies for microsystems fabrication in general, and the development and use of microsystems technologies for microfluidic and biomedical applications in particular. 